\title{
Reactivation of BCG scar as a useful tool in the diagnosis of Kawasaki's disease: A case report
}

\author{
Adeola B Animasahun FACC, FWACP, FMCPAED ${ }^{1}$, Motunrayo O Adekunle FWACP², Olusola Y Kusimo MBBS, MWACP ${ }^{3}$
}

Animasahun AB, Adekunle MO, Kusimo OY. Reactivation of BCG scar as a useful tool in the diagnosis of Kawasaki's disease: A case report. Curr Res Cardiol 2017;4(1):7-8.

Kawasaki disease is self-limiting but can be lethal if there is heart involvement. The diagnostic dilemma that can occur especially in the atypical form can lead to omission and delay in prompt treatment. The reactivation of BCG scar is a clinical manifestation that has been reported in some of the cases and its benefit in regions where BCG vaccine is routinely given has been documented. In Nigeria, there have only been few reported cases of Kawasaki disease and no report on BCG scar reactivation has been done. This case report on a boy with incomplete Kawasaki disease whose diagnosis was further strengthened with the reactivation of BCG scar is reported for its rarity and its benefit in the developing world like ours where BCG vaccination is part of the National Immunization Program schedule.

Key Words: Kawasaki disease; BCG; Scar; Reactivation; Diagnosis
Bacille Calmette-Guérin (BCG) scar reactivation is a clinical manifestation B that can occur in Kawasaki disease and can be a useful addition in making a diagnosis especially in the non-classical type where there is usually a diagnostic dilemma (1).

Bacille Calmette-Guérin vaccine is one of the most widely used vaccines with global coverage rates greater than $80 \%$ in countries endemic for Tuberculosis, including Nigeria where it is included in the National Programme on Immunization schedule (2). It is administered via the intradermal route preferably on the deltoid region of the arm, following which a scar develops within a few months of administration (3).

Bacille Calmette-Guerin inoculation scar reactivation has been reported in Kawasaki disease, occurring in up to $50 \%$ of cases (4) and has been found to be of important diagnostic value (5).

In this present case, the patient had non-classical features of Kawasaki disease but the reactivation of the BCG scar was a strong pointer that guided in the diagnosis and early institution of treatment for Kawasaki disease. With careful literature search, no case of reactivation of BCG scar in Kawasaki disease has been reported in Nigeria. Hence, this case report aims at reporting a case of BCG scar reactivation in a Nigerian child with Kawasaki disease with the hope of not only increasing the awareness of health practitioners on Kawasaki disease but also helping to resolve some diagnostic dilemma that may be faced by practitioners (Figure 1).

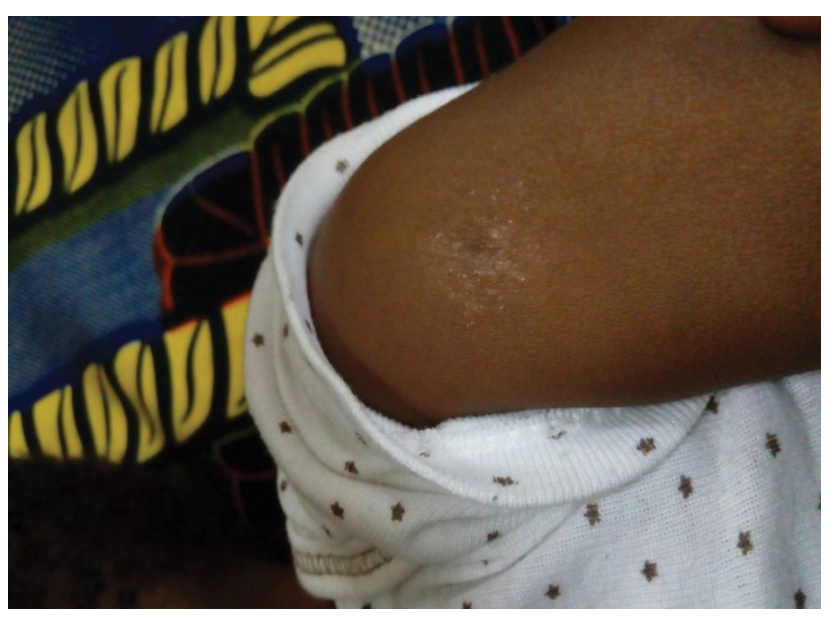

Figure 1) Reactivated BCG scar

\section{CASE REPORT}

A 12-month-old boy who presented with an eight-day history of fever, a fiveday history of bilateral redness of eyes and lips, a four-day history of a trunk rash and a two-day history of bilateral swelling and redness of the hands, feet and a right sided painful neck swelling. Fever was high grade, continuous and only transiently relieved by analgesics administered at home. Fever was not associated with ear discharge or seizures. Bilateral painless redness of the eyes was noticed three days later with no associated eye discharge. There was no history of contact with anyone with red eyes or history suggestive of an upper respiratory tract infection.

Patient's lips were also noticed to have become red and cracked with associated redness of the tongue which resolved before presentation. There was no associated history of bleeding from any other sites, snoring or drooling. $\mathrm{He}$ developed a rash involving the trunk and groin region described as reddish with no associated pruritus. There was no history of contact with anyone with a similar rash and rash resolved prior to presentation at our centre.

Bilateral painful swelling and redness of the hands and feet was noticed two days prior to presentation. Painful right sided neck swelling was noticed at about the same time. The swelling did not progressively increase in size but had resolved about two days prior to presentation at our facility.

He presented in a private hospital about six days after onset of the symptom and was admitted, commenced on intravenous ceftriaxone and antimalarial with no improvement. This prompted a self-referral by parents to our Paediatric Emergency department. His immunization was said to be complete for age according to the National programme on Immunization schedule and BCG scar was sighted.

On presentation, he was irritable but consolable, not in any obvious distress, febrile with temperature of 39.2 degrees Celsius, he had redened, cracked and desquamating lips as well as redness of the oro-pharynx. There was tender non-pitting edema of the hands and feet with associated erythema. Redness of the tongue, bilateral conjunctivitis and desquamation around the perianal region has disappeared at presentation in our center. A diagnosis of a viral exanthem with possibility of Kawasaki disease was considered. However, Crusting and hypopigmentation with some erythema around the BCG scar was later sighted the following day after admission and this further strengthened the possibility of a Kawasaki disease. He later developed periungual desquamation while he was on admission (Figure 2).

Investigations revealed mild anemia with a packed cell volume of $28 \%$, leukocytosis of $17000 / \mathrm{L}$, thrombocytosis with platelets count as high as

${ }^{1}$ Lagos State University College of Medicine, Lagos, Lagos Nigeria, ${ }^{2}$ Babcock University Teaching Hospital, ${ }^{3}$ Lagos University Teaching Hospital

Correspondence: Dr. Adeola B Animasahun, Lagos State University College of Medicine, Lagos, Lagos Nigeria, Telephone +2348037250264, e-mail deoladebo@yahoo.com Received: December 29, 2016, Accepted: March 17, 2017, Published: March 20, 2017

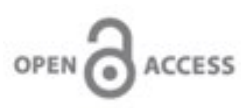

This open-access article is distributed under the terms of the Creative Commons Attribution Non-Commercial License (CC BY-NC) (http:// creativecommons.org/licenses/by-nc/4.0/), which permits reuse, distribution and reproduction of the article, provided that the original work is properly cited and the reuse is restricted to noncommercial purposes. For commercial reuse, contact reprints@pulsus.com 


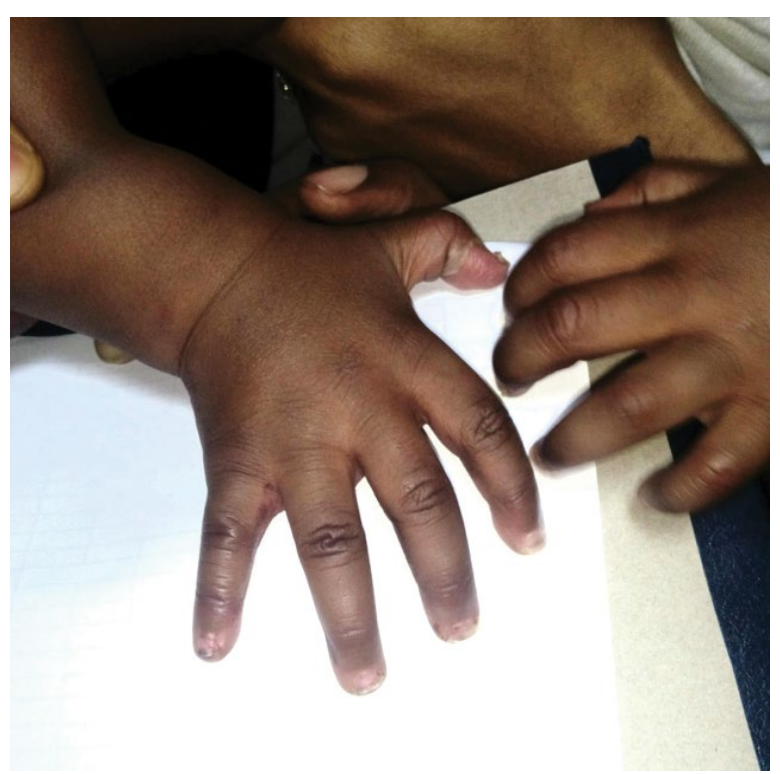

Figure 2) Periungual desquamation

$575,000 / \mathrm{L}$ by the third week of illness. Erythrocyte sedimentation rate was also rose to $131 \mathrm{~mm} /$ hour by the third week of illness, serum albumin was $28 \mathrm{~g} / \mathrm{L}$ and genotype was found to be AA. Serum C3 and C4 and liver function tests were normal. Echocardiography done showed a structurally normal heart.

Intravenous immunoglobulin was administered at $2 \mathrm{~g} / \mathrm{kg}$ and he also received high dose Aspirin at $80 \mathrm{mg} / \mathrm{kg}$, which was reduced to $2 \mathrm{mg} / \mathrm{kg}$ forty-eight hours after the fever had subsided. He had remarkable relief of symptoms before he was discharged.

\section{DISCUSSION}

Kawasaki disease, especially the non-classical form, poses a diagnostic dilemma and at times, not all clinical features required to make the diagnosis are present at the same time (6). In this present case, bilateral redness of the eyes, redness of tongue, a swelling on the cervical region (possibly lymphadenopathy) and desquamation of the skin around the perianal region had disappeared prior to presentation. The manifestation of periungual desquamation later on admission is also in keeping with reported sequential manifestation of symptoms that can occur in Kawasaki disease (6) (Figure 3).

Bacille Calmette-Guerin inoculation scar involvement in Kawasaki disease has been described as crusting, erythema, indurations and even ulceration of a previously otherwise normal BCG scar site (4). This reaction of BCG scar in Kawasaki is possibly due to cross reactivity between Mycobacterial and Human Homologue Heat Shock Protein (5). It occurs in 30 to $50 \%$ cases of Kawasaki disease with a higher prevalence than cervical lymphadenopathy and rash (4). This was a useful finding in this present case where cervical lymphadenopathy and rash were absent at presentation. Crusting with some erythema localized to the area of the BCG scar was a key feature that strengthened the diagnosis of Kawasaki disease in the case reported.

The index patient has been followed up for six months so far. Further follow up is still continued. Possible heart/ coronary manifestation of Kawasaki disease are; congestive heart failure, left ventricular dysfunction, myocarditis, pericarditis, valvular regurgitation (aortic and mitral), aortic root dilatation, coronary artery abnormalities including aneurysms of medium-size non coronary arteries. Coronary artery aneurysm is the gravest and most important complication of Kawasaki disease (7).

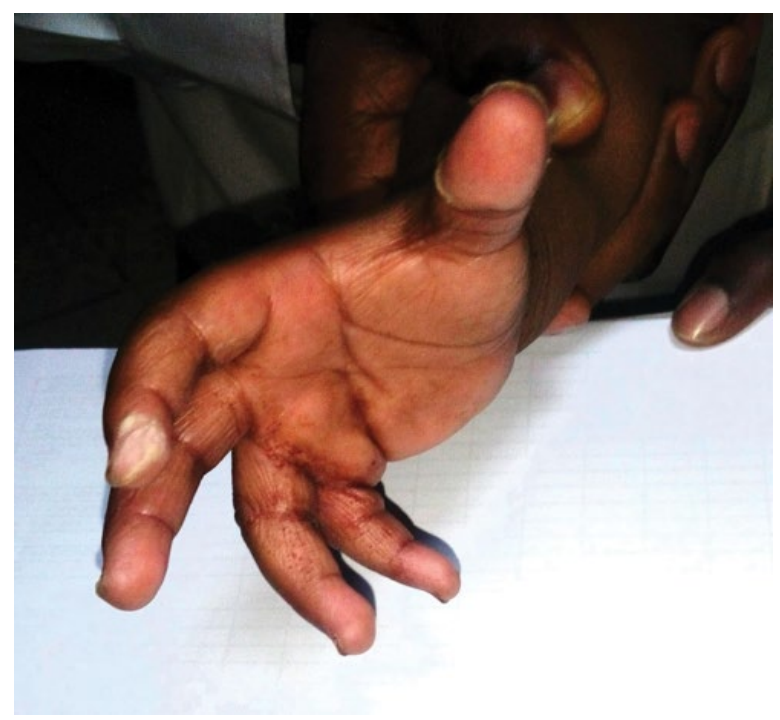

Figure 3) Periungual desquamation

\section{CONCLUSION}

Kawasaki disease is clinically diagnosed and has an increased mortality in cases with coronary involvement and delay in the institution of therapy. There is often a diagnostic dilemma especially in incomplete cases where heart involvement can also occur. Reactivation of BCG scar is of benefit in making a diagnosis of Kawasaki disease especially in places where BCG vaccination is part of the National Immunization Program.

\section{ACKNOWLEDGEMENT}

The authors acknowledge the subject, his parent, other healthcare practitioners who participated in his care.

\section{DISCLOSURES}

None.

\section{ETHICAL DISCLOSURES}

Informed and written consent were obtained from the parent of this reported case. The necessary institutional protocols on the publication of the data were duly followed.

\section{CONFLICT OF INTEREST}

No conflict of interest among the authors.

\section{REFERENCES}

1. Burns JC, Glode MP. Kawasaki syndrome. Lancet 2004;364:533-44.

2. Nakamura Y, Yashiro A, Uehara R, et al. Epidemiologic features of Kawasaki disease in Japan: results of the 2009-2010 nationwide survey. J Epidemiol 2012;22(3):210-21.

3. Nakamura Y, Yanagawa H. The worldwide epidemiology of Kawasaki disease. Prog Pediatr Cardiol 2004;19:99-08.

4. Ikpatt NW, Ibia EO. Mucocutaneous lymph node syndrome in CalabarA case report. East Afr Med J. 1989;66(11):776-81.

5. Sotimehin SA, Ogunlesi TA, Adekanmbi AF, et al. Kawasaki disease in a Nigerian child - A case report. Nigerian Medical Practitioner 2010;57(4).

6. Sani UM, Ahmed H. Kawasaki disease: An unusual presentation in a 14-year old boy in Sokoto, north western Nigeria. Niger J Paed 2013;40(4):422-25.

7. Mc Crindle BN, Li JS, Minich LL, et al. Coronary artery involvement in children with Kawasaki disease. Risk factors from analysis of serial normalized measurements. Circulation 2007:116:174-79. 